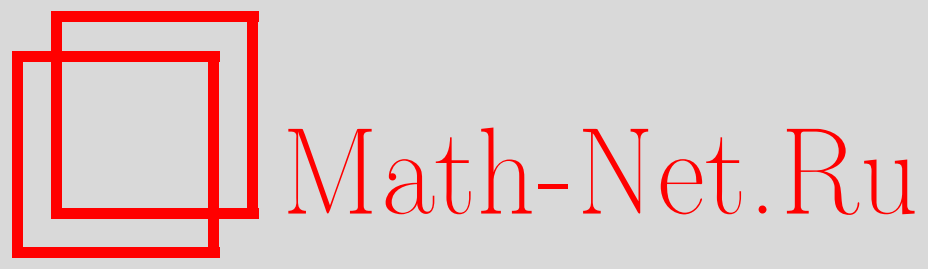

Е. А. Горин, Д. В. Трещёв, Относительный вариант теоремы Титчмарша о свертке, Функи. анализ и его прил., 2012, том 46, выпуск 1, 31-38

DOI: https://doi.org/10.4213/faa3052

Использование Общероссийского математического портала MathNet.Ru подразумевает, что вы прочитали и согласны с пользовательским соглашением

http://www . mathnet.ru/rus/agreement

Параметры загрузки:

IP : 54.198 .55 .26

26 апреля 2023 г., 11:49:03

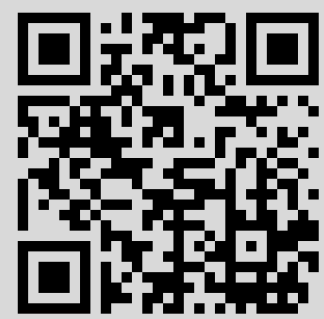




\title{
Относительный вариант теоремы Титчмарша о свертке*
}

\begin{abstract}
Рассматривается алгебра $C_{u}=C_{u}(\mathbb{R})$ всех равномерно непрерывных ограниченных комплексных функций на вещественной оси $\mathbb{R}$ с поточечными операциями и sup-нормой. Пусть $I-$ замкнутый идеал в $C_{u}$, инвариантный относительно сдвигов. Обозначим через $\mathrm{ah}_{I}(f)$ наименьшее вещественное число (если оно существует), удовлетворяющее следующему условию: если $\lambda>\mathrm{ah}_{I}(f)$, то $\left.(\hat{f}-\hat{g})\right|_{V}=0$ для некоторого $g \in I$, где $V$ - окрестность точки $\lambda$. Классическая теорема Титчмарша о свертке равносильна равенству $\mathrm{ah}_{I}\left(f_{1} \cdot f_{2}\right)=\mathrm{ah}_{I}\left(f_{1}\right)+\mathrm{ah}_{I}\left(f_{2}\right)$, где $I=\{0\}$. Устанавливается, что для идеалов $I$ общего вида указанное равенство, как правило, места не имеет, но равенство $\operatorname{ah}_{I}\left(f^{n}\right)=n \cdot \operatorname{ah}_{I}(f)$ справедливо для любого $I$. В то же время представлено много нетривиальных идеалов, для которых общая форма теоремы Титчмарша верна.
\end{abstract}

1. Классическая теорема Титчмарша о свертке устанавливает, что для пары суммируемых функций на вещественной оси $\mathbb{R}$ с компактными носителями выпуклая оболочка носителя свертки совпадает с арифметической суммой выпуклых оболочек носителей исходных функций. Она легко распространяется на распределения Шварца над пространством $S=S(\mathbb{R})$ быстро убывающих функций и имеет многомерные варианты. В некоторой форме, которую мы напомним ниже, теорема о свертке распространяется на функции, сосредоточенные на полуосях, направленных в одну и ту же сторону. Впрочем, большинство обобщений сводятся к тому случаю, когда обе функции суть гладкие функции с компактными носителями (правда, подобная редукция может и скрыть простую суть дела).

В работе [1] при изучении финальной динамики в системе взаимодействующих осциллятора и термостата потребовался «относительный вариант» теоремы Титчмарша, в котором при определении дополнения к носителю локальное совпадение с нулевой функцией заменяется локальной принадлежностью некоторому идеалу (в дальнейшем мы уточним формулировки). Цель данной работы - придать математическим результатам из [1] более простую и общую форму. В принципе здесь можно было бы говорить о «сингулярных носителях», подобно тому, как это делается в теории распределений, когда говорят об отклонении от гладкости или аналитичности, однако с алгебраической точки зрения, которой мы здесь придерживаемся, уместнее говорить об «относительных» вариантах.

Теорему Титчмарша при всей очевидности ее дискретных случаев, когда берутся, например, конечные линейные комбинации сдвигов $\delta$-функции Дирака, никак нельзя отнести к числу тривиальных. Эта теорема имеет многочисленные применения, и с годами было найдено много ее разнообразных доказательств и обобщений. На наш взгляд, одним из самых прозрачных является доказательство, приведенное в лекциях Б. Я. Левина [2, лекция 16]. Правда, оно использует

* Исследование второго автора выполнено при частичной поддержке программы Президиума Российской академии наук «Математическая теория управления». 
не только классические (начала прошлого века) факты теории аналитических функций, но и такие, которые были открыты позже теоремы Титчмарша. Вместе с тем, с небольшими модификациями, близкая схема работает и в той ситуации, которая нас интересует, хотя дословно перенести рассуждение нельзя (просто потому, что относительный вариант теоремы о носителе свертки дословно выполняется не в полном объеме).

2. Пусть $M=M(\mathbb{R})$ - комплексная банахова алгебра всех комплексных (регулярных) борелевских мер конечной полной вариации на $\mathbb{R}$. B качестве нормы берется полная вариация, а в качестве умножения - свертка, которая определяется равенством

$$
\int_{\mathbb{R}} g(t)\left(\mu_{1} * \mu_{2}\right)(d t)=\int_{\mathbb{R} \times \mathbb{R}} g(s+t) \mu_{1}(d s) \mu_{2}(d t) .
$$

В определении свертки мер это равенство должно выполняться для всех непрерывных функций $g$, «исчезающих на бесконечности», но тогда оно выполняется и для всех ограниченных непрерывных функций (детальное обсуждение этой и других банаховых алгебр, а также фундаментальных фактов гармонического анализа можно найти, например, в [3]).

Если использовать стандартное спаривание $\langle g, \mu\rangle=\int_{\mathbb{R}} g(t) \mu(d t)$ и положить $(g \star \mu)(x) \stackrel{\text { def }}{=} \int_{\mathbb{R}} g(x+t) \mu(d t)$, то определение свертки мер можно будет представить в виде $\left\langle g, \mu_{1} * \mu_{2}\right\rangle=\left\langle g \star \mu_{1}, \mu_{2}\right\rangle$.

Мы будем также использовать и обозначение $(g * \mu)(x) \stackrel{\text { def }}{=} \int_{\mathbb{R}} g(x-t) \mu(d t)$, которое согласовано со всеми стандартными.

Преобразование Фурье меры $\mu$ - это функция на двойственной оси, определяемая равенством

$$
\widehat{\mu}(\lambda)=\int_{\mathbb{R}} e^{i \lambda t} \mu(d t) .
$$

Через $M_{0}=M_{0}(\mathbb{R})$ мы будем обозначать совокупность мер $\mu \in M$, абсолютно непрерывных по мере Лебега. Хорошо известно (и легко проверить), что $M_{0}-$ замкнутый идеал в $M$.

Каждая мера $\mu \in M_{0}$ имеет плотность $h \in L^{1}(\mathbb{R})\left(L^{1}(\mathbb{R})\right.$ - пространство Лебега классов эквивалентности суммируемых функций). Обычно плотности отождествляют с мерами, и это позволяет говорить о свертках, преобразованиях Фурье и тому подобных операциях с участием $L^{1}$-функций (в свое время только такие меры и рассматривали, без конца повторяя «почти всюду»). Мы также будем использовать «плотности» (и говорить об отвечающих им функциях, обычно гладких), не забывая, однако, что с формальной точки зрения в подобных случаях речь идет о мерах. Разумеется, термин «функция», как обычно, используется и в более широком смысле.

Каждый из функционалов $\mu \rightarrow \widehat{\mu}\left(\lambda_{0}\right)$ задает непрерывный сохраняющий единицу гомоморфизм из $M$ в поле $\mathbb{C}$ комплексных чисел. Ядро такого функционала - максимальный идеал, но, как известно, такими идеалами не исчерпывается даже граница Шилова пространства максимальных идеалов. Вместе с тем, для $\widehat{M}_{0}$ нет других гомоморфизмов в $\mathbb{C}$, кроме точечных. Кроме того, $\widehat{M}$ и $\widehat{M}_{0}$ локально изоморфны (как алгебры функций на двойственной оси).

3. Пусть $C_{u}=C_{u}(\mathbb{R})$ - алгебра всех равномерно непрерывных ограниченных комплексных функций на вещественной оси. Относительно поточечных операций и sup-нормы $C_{u}$ является $C^{*}$-алгеброй и в таком качестве, согласно 
теореме Гельфанда-Наймарка, изометрически изоморфна алгебре всех непрерывных функций на некотором компакте. Этот компакт является компактным (= бикомпактным) расширением ${ }^{1)}$ вещественной оси и обозначается здесь через $\alpha \mathbb{R}$ (с целью подчеркнуть, что оно близко к чеховскому расширению $\beta \mathbb{R}$, но подчиняется ему). Как и во всех $C^{*}$-алгебрах, каждый замкнутый идеал в $C_{u}$ симметричен.

Положим $\left(T_{x} f\right)(t)=f(x+t)$. Выбор алгебры $C_{u}$ вместо алгебры $C_{b}$ всех ограниченных непрерывных функций объясняется тем, что в случае $C_{u}$ представление $x \rightarrow T_{x}$ является сильно непрерывным, т. е. при каждом фиксированном $f$ функция $x \rightarrow T_{x} f$ непрерывна.

Пространство $C_{u}$ вместе с векторной структурой и нормой естественно погружается в сопряженное к $M_{0}$. Поэтому мы можем определить преобразование Фурье $\widehat{g}$, считая его функционалом над $\widehat{M}_{0}$,

$$
\langle\widehat{g}, \widehat{\mu}\rangle=2 \pi\langle g, \mu\rangle .
$$

Заметим, что топология на $\widehat{M}_{0}$ переносится с $M_{0}$.

Аналогичное определение используется в теории распределений. Так как $S \subset M_{0}$, то эти определения согласованы. Таким образом, привлекая (или нет) теорию распределений, мы получаем возможность говорить (и судить) о локальных свойствах преобразований Фурье функций из $C_{u}$.

4. При $\sigma>0$ через $\mathbf{B}_{\sigma}$ обозначается пространство Бернштейна, которое включает все целые функции экспоненциального типа $\leqslant \sigma$, ограниченные на вещественной оси. Относительно sup-нормы (по $\mathbb{R}$ ) это пространство является банаховым. Согласно неравенству Бернштейна ${ }^{2)}$,

$$
|f(x+h)-f(x)| \leqslant 2 \sin (\sigma h / 2) \cdot \sup _{\mathbb{R}}|f|, \quad 0<\sigma h<\pi,
$$

для всех $f \in \mathbf{B}_{\sigma}$.

Пусть $\mathbf{B}=\bigcup_{\sigma>0} \mathbf{B}_{\sigma}$. Ясно, что $\mathbf{B} \subset C_{u}$. Более того, по (другой) теореме Бернштейна $\mathbf{B}$ всюду плотно в $C_{u}$. Для доказательства этой теоремы удобно рассмотреть свертки $f_{\rho}=f * v_{\rho}$, где $\left\{v_{\rho}\right\}$ - «ядро Валле Пуссена», $\rho>0$. Функция $\widehat{v}_{\rho}$ сосредоточена на отрезке $|\lambda| \leqslant 2 \rho$, равна 1 при $|\lambda| \leqslant \rho$ и линейна на двух остальных промежутках.

Из равномерной непрерывности легко вытекает равномерная сходимость $f_{\rho} \rightarrow f$ при $\rho \rightarrow \infty$. Кроме того, $\hat{f}_{\rho}=\hat{f}$, если $|\lambda|<\rho$. Наконец, если $I-$ инвариантный относительно сдвигов идеал в алгебре (по умножению) $C_{u}$ и $f \in I$, то $f_{\rho} \in I$ при каждом $\rho>0$.

Перечисленные факты, как отмечалось, позволяют упростить некоторые доказательства, однако в ряде случаев без них лучше обойтись.

5. Предположим, что $f$ - непрерывная функция в замкнутой верхней полуплоскости $y=\operatorname{Im} z \geqslant 0$ комплексной плоскости $\mathbb{C}$, аналитическая при $y>0$. Если $f$ ограничена на вещественной оси $\mathbb{R}$ и имеет экспоненциальный тип

1) Такие расширения в середине прошлого века систематически использовал Ю. М. Смирнов для изучения в рамках общей топологии введенных В. А. Ефремовичем пространств близости.

2) Предъявленное неравенство означает, что норма оператора сдвига в $\mathbf{B}_{\sigma}$ совпадает со спектральным радиусом. Когда происходит подобное совпадение для операторов, далеких от нормальных в гильбертовом пространстве, объяснялось еще в [4]. 
$\sigma>0$ в верхней полуплоскости ${ }^{1)}$, то, согласно классической теореме ФрагменаЛинделёфа,

$$
|f(z)| \leqslant e^{\sigma y} \sup _{\mathbb{R}}|f| .
$$

Следующее асимптотическое равенство (см. [2, лекция 16]) дополняет и в известном смысле уточняет приведенное неравенство:

$$
\log |f(z)|=\sigma y+o(y) .
$$

Оно выполняется на почти всех лучах $0<\arg z<\pi$ и на каждом луче, если из него удалить множество конечной логарифмической длины.

Напомним, что измеримое множество $E$ по определению имеет конечную логарифмическую длину на полуоси $r>0$, если $\int_{E}(1+r)^{-1} d r<\infty$.

Заметим, что соотношение (2) остается справедливым при сравнительно небольшом росте функции $f$ на вещественной оси, в частности, если рост не выше степенного.

6. Пусть $f \in C_{u}$ и носитель $\operatorname{supp}(\hat{f})$ преобразования Фурье функции $f$ расположен на некоторой полуоси $\lambda \leqslant \lambda_{0}<\infty$. Наименьшее из таких $\lambda_{0}$ обозначается через $\mathrm{ah}(f)$ и называется гармонической абсииссой функции $f$. Если конечных $\lambda_{0}$ с указанным свойством нет, то считается, что $\operatorname{ah}(f)=+\infty$. Следующая лемма (доказательство которой мы опустим) выводится при помощи стандартных аналитических средств (включая соотношение (2)).

Лемма 1. Пусть $f \in C_{u} u \sigma>0$. Условие $\mathrm{ah}(f) \leqslant \sigma$ выполняется тогда u только тогда, когда продолэсение по Пуассону функиии $x \rightarrow f(x) e^{i \sigma x}$ в полуплоскость $\operatorname{Im} z>0$ является там ограниченной аналитической функиией. Кроме того, если $0<\operatorname{ah}(f)<\infty$, то

$$
\operatorname{ah}(f)=\lim _{y \rightarrow \infty}^{*} y^{-1} \log |f(i y)|,
$$

где звездочка означает, что предел (совпадающий с верхним) существует, если из у-оси предварительно удалить подходящее множсество конечной логарифмической длинъ.

Пусть $f \in C_{u}$, причем $\operatorname{ah}(f)<\infty$. Положим $\left(T_{z} f\right)(t)=f(z+t)$. Ясно, что $T_{z} f \in C_{u}$. Следующая лемма вытекает из предыдущей (и доказательство мы снова опустим).

Лемма 2. Фиксируем такую функиию $f \in C_{u}$, что $\operatorname{ah}(f)<\infty$. Тогда функиия $z \rightarrow T_{z} f$ является непрерьвной функиией в замкнутой верхней полуплоскости $\operatorname{Im} z \geqslant 0$ со значениями в $C_{u}$, аналитической при $\operatorname{Im} z>0$.

Из формулы (3) сразу вытекает равенство

$$
\operatorname{ah}\left(f_{1} \cdot f_{2}\right)=\operatorname{ah}\left(f_{1}\right)+\operatorname{ah}\left(f_{2}\right) \quad\left(\operatorname{ah}\left(f_{1}\right), \operatorname{ah}\left(f_{2}\right)<\infty\right),
$$

эквивалентное классической теореме Титчмарша.

7. Пусть $f \in C_{u}$, и пусть $I-$ замкнутый идеал в $C_{u}$, инвариантный относительно сдвигов. Тогда $f * \mu \in I$ для каждой меры $\mu \in M$, и мы будем этим широко пользоваться.

Рассмотрим такие точки $\lambda_{0} \in \mathbb{R}$ (здесь $\mathbb{R}$ - двойственный экземпляр вещественной оси), что в некоторой окрестности точки $\lambda_{0}$ распределение $\hat{f}$ совпадает

1) К выполнению условия $\sigma>0$ приводит (при необходимости) вещественный сдвиг функции $f$, так что оно «не снижает общности», однако заметно упрощает некоторые формулировки и рассуждения. 
с $\widehat{g}$, где $g \in I$. Легко видеть, что (как и в тривиальном случае) множество всех таких точек $\lambda_{0}$ открыто. Дополнение к этому открытому множеству (на двойственной оси) естественно обозначить через $\operatorname{supp}_{I}(\hat{f})$. Иногда мы будем называть его относительным гармоническим носителем функции $f$. В частности, (абсолютный) гармонический носитель - это $\operatorname{supp}(\hat{f})$. Заметим, что с позиций классического гармонического анализа гармонический носитель - это спектр Бьёрлинга, а с позиций теории Гельфанда-Шилова - это оболочка $\operatorname{hull}(K)$ идеала $K=\operatorname{Ann}(f)$ (= аннулятора функционала $f$ в алгебре суммируемых функций по свертке).

В дальнейшем, если противное не оговаривается (как теперь говорят, «по умолчанию»), мы рассматриваем функции $f \in C_{u}$, для которых $\operatorname{ah}(f)<\infty$. То же относится к предположению об инвариантности идеалов. С другой стороны, напоминание этих обстоятельств никакой дополнительной смысловой нагрузки не несет.

Относительная гармоническая абсиисса $\mathrm{ah}_{I}(f)$ - это наименьшее число, ограничивающее сверху $\operatorname{supp}_{I}(\hat{f})$. Ясно, что $\operatorname{ah}_{I}(f) \leqslant \operatorname{ah}(f)$ для всех идеалов $I$.

Наша задача - выяснить, в какой мере соотношение (4) распространяется на относительные гармонические абсциссы. Отметим сразу, что оно, вообще говоря, нарушается, причем в очень простых случаях.

8. Расширению $\alpha \mathbb{R}$ вместе с топологией фактически сопоставляются гомоморфизмы $\varphi: C_{u} \rightarrow \mathbb{C}$. Каждому замкнутому идеалу $I \subset C_{u}$ однозначно соответствует такое замкнутое множество $Q \subset \alpha \mathbb{R}$, что $I=\{f: \varphi(f)=0$ для всех $\varphi \in Q\}$. Более того, факторалгебра (естественно) изометрически изоморфна алгебре $C(Q)$ с sup-нормой. Норма образа элемента $f \in C_{u}$ в образе $C_{u} \rightarrow C_{u} / I$ обозначается через $\|f\|_{C_{u} / I}$. С алгебраической точки зрения $Q=\operatorname{hull}(I)-a \Omega$ гебраическая оболочка идеала $I$.

Операторы $T_{x}^{*}$, сопряженные к автоморфизмам $T_{x}$, естественно порождают гомеоморфизмы компакта $\alpha \mathbb{R}$, так что $\left(T_{x}^{*} \varphi\right)(f)=\varphi\left(T_{x} f\right)$. Инвариантность идеала $I=I(Q)$ относительно сдвигов равносильна тому, что $\varphi$ и $T_{x}^{*} \varphi$ одновременно принадлежат $Q$ или нет.

Легко проверить, что описанное действие $\mathbb{R}$ на $\alpha \mathbb{R}$ является свободным.

Если $\varphi$ - гомоморфизм из $C_{u}$ в $\mathbb{C}$, то через $Q_{\varphi}$ мы будем обозначать замыкание орбиты $\left\{T_{x}^{*} \varphi\right\}$. Если $\varphi$ отвечает точке из $\mathbb{R}$, то $Q_{\varphi}=\alpha \mathbb{R}$. В противном случае $Q_{\varphi} \cap \mathbb{R}=\varnothing$.

Следующая лемма - один из основных результатов.

Лемма 3. Пусть $I \subset C_{u}-$ инвариантный замкнутый идеал, и пусть $f \in$ $C_{u}$ - бункиия с полуограниченным справа гармоническим спектром. Тогда

$$
\operatorname{ah}_{I}(f)=\varlimsup_{y \rightarrow \infty} y^{-1} \log \left\|T_{i y} f\right\|_{C_{u} / I} .
$$

Доказательство. Обозначим через $\alpha$ левую часть в (5) и через $\beta$ - правую. Не ограничивая общности, можно считать, что оба эти числа положительны. Заметим, что тогда и $\operatorname{ah}(f)>0$.

Отметим, что если $I=\{0\}$, то лемма 3 фактически покрывается леммой 1.

Покажем сначала, что $\beta \leqslant \alpha$.

Пусть $\varepsilon>0$. Используя соображения вроде указанных в разд. 4, можно найти такую функцию $g \in \mathbf{B} \cap I$, зависящую только от $f$ и $\varepsilon$, что $\operatorname{ah}(f-g)<\alpha+\varepsilon$. Далее, $T_{i y} g \in I$. Действительно, например, для функций $g$, ограниченных в 
верхней полуплоскости, «сдвиг вверх» дает свертка с ядром Пуассона, а свертка оставляет в идеале, так как он инвариантен относительно (вещественных) сдвигов. Таким образом,

$$
\left\|T_{i y} f\right\|_{C_{u} / I}=\left\|T_{i y}(f-g)\right\|_{C_{u} / I} \leqslant\left\|T_{i y}(f-g)\right\| \leqslant \operatorname{const} \cdot e^{(\alpha+\varepsilon) y},
$$

так что $\beta \leqslant \alpha$.

Допустим теперь, что $\beta+\varepsilon<\alpha$ при некотором $\varepsilon>0$, и покажем, что это приводит к противоречию.

Выберем такую функцию $h \in S$, что

$$
\widehat{h}(\lambda)= \begin{cases}1, & \text { если }|\lambda-\alpha| \leqslant \varepsilon / 2, \\ 0, & \text { если }|\lambda-\alpha| \geqslant \varepsilon\end{cases}
$$

При таком выборе заведомо

$$
f * h \notin I \text {. }
$$

Пусть $\psi$ - такой непрерывный линейный функционал на $C_{u}$, что $\psi \mid I=0$ и $\|\psi\|=1$. При $y \geqslant 0$ положим $g(z)=\psi\left(T_{z} f\right)$. Эта функция непрерывна при $\operatorname{Im} z \geqslant 0$, ограничена и равномерно непрерывна на каждой горизонтальной оси в замкнутой верхней полуплоскости и является аналитической при $\operatorname{Im} z>0$ (лемма 2).

Так как $\psi \mid I=0$, то $|g(i y)| \leqslant$ const $\cdot e^{\beta y}$ при $y \geqslant 0$. Поэтому $\operatorname{ah}(g) \leqslant \beta$. Следовательно, $g * h=0$. Таким образом,

$$
0=(g * h)(0)=\int_{\mathbb{R}} \psi\left(T_{-x} f\right) h(x) d x=\psi\left(\int_{\mathbb{R}}\left(T_{-x} f\right) h(x) d x\right)=\psi(f * h) .
$$

Так как $\psi$ - произвольный функционал (удовлетворяющий указанным условиям), то по теореме Хана-Банаха непременно $f * h \in I$, что, однако, противоречит условию (6), и лемма доказана.

В качестве очевидного следствия мы получаем неравенство

$$
\operatorname{ah}_{I}\left(f_{1} \cdot f_{2}\right) \leqslant \operatorname{ah}_{I}\left(f_{1}\right)+\operatorname{ah}_{I}\left(f_{2}\right) .
$$

Приведем простой пример, который показывает, что неравенство (7) может оказаться строгим. Выделим однозначную аналитическую ветвь функции $\sqrt{z}$ в верхней полуплоскости условием $\sqrt{1}=1$. Положим $f_{1}(z)=\exp (-\sqrt{z})$. Через $f_{2}$ обозначим аналогичную функцию в верхней полуплоскости с убыванием к 0 на отрицательной полуоси. Пусть $C_{0}$ - идеал функций, для которых на вещественной оси $|f(x)| \rightarrow 0$ при $|x| \rightarrow \infty$. Очевидно, что $f_{1} f_{2} \in C_{0}$, хотя ни один из сомножителей $C_{0}$ не принадлежит.

Вместе с тем, для совпадающих сомножителей равенство сохраняется (и сразу вытекает из леммы 3):

Теорема 1. $\operatorname{ah}_{I}\left(f^{n}\right)=n \cdot \operatorname{ah}_{I}(f)$.

Замечание. Общий случай теоремы 1 стандартным способом сводится к случаю $n=2$. Действительно, тогда по индукции теорема выполняется для всех $n=2^{k}$. Далее, при других $n$, выбирая $m$ из условия $2^{n}=m+n$ и используя неравенство (7) (которое, кстати, легко доказать непосредственно), мы получим теорему 1 в общем случае.

Любопытно, что в классической ситуации, имеются пути (впервые обнаруженные Микусинским), позволяющие, отправляясь от квадратов, перейти к 
произведениям произвольных пар (см., например, [5, п. 4.3]). Приведенный выше пример, в частности, показывает, что в нашем случае такой «скачок» невозможен.

9. В доказательстве леммы 3 мы использовали все непрерывные функционалы $\psi$, ядро которых содержит идеал $I$, хотя могли бы привлекать только мультипликативные, поскольку каждый замкнутый идеал в $C_{u}$ - пересечение максимальных. В приводимой ниже лемме, аналогичной лемме 3, такое сужение оказывается принципиально важным. Более того, формально говоря, нам хватит всего лишь одного мультипликативного функционала.

Напомним, что при $\varphi \in \alpha \mathbb{R}$ через $Q_{\varphi}$ мы обозначаем замыкание орбиты «точки» $\varphi$. Ниже имеет смысл сразу предположить, что $\varphi \notin \mathbb{R}$. Как и в лемме 3 , мы считаем, что $\mathrm{ah}(f)<\infty$. Символы $\alpha$ и $\beta$ имеют тот же смысл, что и в лемме 3 , а символ $\lim ^{*}$ - тот же смысл, что и в лемме 1.

Лемма 4. Если $f \in I=I\left(Q_{\varphi}\right)$, mo

$$
\operatorname{ah}_{I}(f)=\lim _{y \rightarrow \infty}^{*} y^{-1} \log \left|\varphi\left(T_{i y} f\right)\right|
$$

Доказательство. Обозначим через $\gamma$ правую часть в (8). Очевидно, что $\gamma \leqslant \beta$. Так как, согласно лемме $3, \alpha=\beta$, то достаточно проверить, что $\beta \leqslant \gamma$.

Заметим, что для подалгебры функций $f \in C_{u}$ c $\operatorname{ah}(f)<\infty$ при фиксированном $z$ из верхней полуплоскости отображение $f \rightarrow T_{z} f$ является эндоморфизмом и, следовательно, $f \rightarrow \varphi\left(T_{z} f\right)$ - гомоморфизмом в $\mathbb{C}$ (в условиях леммы 4 предположение $\mathrm{ah}(f)<\infty$ считается выполненным). Наконец, ясно, что

$$
\|f\|_{C_{u} / I}=\sup _{x \in \mathbb{R}}\left|\left(T_{x}^{*} \varphi\right)(f)\right| .
$$

Предположим, что $\|f\|=1$ (это не меняет дела). Пусть по определению $g(z)=\varphi\left(T_{z} f\right)$ при $\operatorname{Im} z \geqslant 0$. Функция $g$ имеет конечный экспоненциальный тип в верхней полуплоскости, причем $|g(x)| \leqslant 1$ на вещественной оси. Поэтому по формуле (1) (т. е. по теореме Фрагмена-Линделёфа) $|g(z)| \leqslant \exp \gamma y$.

Последнее неравенство вместе с формулой (9) приводит к неравенству $\beta \leqslant \gamma$, и лемма доказана.

Теорема 2. Если $I=I\left(Q_{\varphi}\right)$, mо

$$
\operatorname{ah}_{I}\left(f_{1} \cdot f_{2}\right)=\operatorname{ah}_{I}\left(f_{1}\right)+\operatorname{ah}_{I}\left(f_{2}\right) .
$$

Заметим, что, формально говоря, теорема 2 включает теорему Титчмарша.

Пусть $Q_{1}, Q_{2}$ - инвариантные замкнутые подмножества в $\alpha \mathbb{R}$ и $Q=Q_{1} \cup Q_{2}$. Следующая теорема - общая форма приведенного выше примера.

Теорема 3. Если $Q_{1} \backslash Q_{2} \neq \varnothing \neq Q_{2} \backslash Q_{1}$, то для идеала $I=I(Q)$ соотношение (10), вообще говоря, нарушается.

Доказательство. В дальнейшем $k=1$ или 2. Пусть $\varphi_{k} \in Q \backslash Q_{k}$. Существуют такие функции $f_{k}$, что $f_{k} \in I\left(Q_{k}\right)$ и $\varphi_{k}\left(f_{k}\right)=1$. Из разд. 4 вытекает существование таких функций $g_{k} \in \mathbf{B} \cap I\left(Q_{k}\right)$, что $\left\|f_{k}-g_{k}\right\|<\varepsilon<1 / 2$. Обе функции $g_{1}, g_{2}$ имеют компактные гармонические спектры и обе не принадлежат $I$, так что их гармонические абсциссы - конечные вещественные числа. Вместе с тем $g_{1} \cdot g_{2} \in I$, и теорема доказана.

10. Нам осталось сделать несколько замечаний. Вероятно, теоремы 2 и 3 допускают синтез, приводящий к критерию, т.е. к описанию всех идеалов, для 
которых теорема Титчмарша выполняется в полном объеме. Естественно предположить, что для достижения этой цели потребуется гораздо более тщательное изучение «пространства орбит».

Очевидно, что такой критерий, как и теорема 1, представляют интерес и в многомерном случае. Для решения многомерных задач представляется перспективной попытка распространить на нашу ситуацию первоначальный подход Лионса [6]-[8], более современное описание которого см., например, в [9, п. 16.3]. Не исключено, что применение указанного выше асимптотического соотношения (2) может оказаться полезным и, вероятно, приведет к некоторым упрощениям даже в классической ситуации. Конечно, многомерные варианты интересны и для теоремы 1. Более того, для классической теоремы Титчмарша известно, в каких топологических абелевых группах она выполняется (см., в частности, [10], [11]), скажем, годится группа $\mathbb{R}^{n}$, но взятая в дискретной топологии. Аналогичный вопрос имеет смысл и в нашем случае.

Мы благодарим А. Б. Александрова, строгая, но справедливая критика которого первоначального варианта заставила превратить текст в гораздо более внятный.

\section{ЛитературА}

[1] D. V. Treschev, Oscillator and thermostat, Discrete Contin. Dyn. Syst. (DCDS-A), 28:4 (2010), 1693-1712 .

[2] B. Ya. Levin, Lectures on Entier Functions, Translations of Math. Monographs, vol. 150, Amer. Math. Soc., Providence, RI, 1996.

[3] Н. Бурбаки, Спектральная теория, Мир, М., 1972.

[4] Е. А. Горин, Об исследованиях Г. Е. Шилова по теории коммутативных банаховых алгебр и их дальнейшем развитии, УМН, 33:4 (1978), 169-188.

[5] Л. Хёрмандер, Анализ линейных дифференииалъных операторов с частными производными, m. 1. Теория распределений и анализ Фурье, Мир, М., 1986.

[6] J. L. Lions, Supports de produits de compositions, C.R. Acad. Sci., 232 (1951), 15301532.

[7] J. L. Lions, Supports de produits de compositions. I, C.R. Acad. Sci., 232 (1951), $1622-1624$.

[8] J. L. Lions, Supports dans la transfomations de Laplace. II, J. Anal. Math., 2 (1953), 369-380.

[9] Л. Хёрмандер, Анализ линейных дифференциальных операторов с частными производными, т. 2. Дифференциальные операторы с постоянными коэффициентами, Мир, М., 1986.

[10] B. Weiss, Titcmarsh's convolutions theorem on groups, Proc. Amer. Math. Soc., 19 (1968), 75-79.

[11] Y. Domar, Convolutions theorem of Titchmarsh type on discrete $\mathbf{R}^{n}$, Proc. Edinburgh Math. Soc., 32:3 (1989), 449-457.

Московский педагогический государственный университет e-mail: evgeny.gorin@mtu-net.ru Поступило в редакцию 28 марта 2011 г.

Институт математики РАН им. В. А. Стеклова e-mail: treschev@mi.ras.ru 